\title{
Development and optimisation of low-power magnetic flux leakage inspection parameters for mild steel welds
}

DOI:

10.1784/insi.2021.63.2.75

\section{Document Version}

Accepted author manuscript

Link to publication record in Manchester Research Explorer

\section{Citation for published version (APA):}

Watson, J. M., Liang, C. W., Sexton, J., \& Missous, M. (2021). Development and optimisation of low-power magnetic flux leakage inspection parameters for mild steel welds. Insight: Non-Destructive Testing and Condition Monitoring, 63(2), 75-81. https://doi.org/10.1784/insi.2021.63.2.75

\section{Published in:}

Insight: Non-Destructive Testing and Condition Monitoring

\section{Citing this paper}

Please note that where the full-text provided on Manchester Research Explorer is the Author Accepted Manuscript or Proof version this may differ from the final Published version. If citing, it is advised that you check and use the publisher's definitive version.

\section{General rights}

Copyright and moral rights for the publications made accessible in the Research Explorer are retained by the authors and/or other copyright owners and it is a condition of accessing publications that users recognise and abide by the legal requirements associated with these rights.

\section{Takedown policy}

If you believe that this document breaches copyright please refer to the University of Manchester's Takedown Procedures [http://man.ac.uk/04Y6Bo] or contact uml.scholarlycommunications@manchester.ac.uk providing relevant details, so we can investigate your claim.

\section{OPEN ACCESS}




\title{
Development and Optimisation of Low Power Magnetic Flux Leakage Inspection Parameters for Mild Steel Welds
}

\author{
J. M. Watson AMInstNDT, C.W. Liang, J. Sexton and M. Missous \\ University of Manchester, Department of Electrical and Electronic Engineering \\ Manchester, M13 9PL, United Kingdom \\ james.watson-6@postgrad.manchester.ac.uk
}

\begin{abstract}
Magnetic particle and other magnetic flux leakage (MFL) based methods for the detection and evaluation of surface-breaking flaws in ferromagnetic materials typically use high-strength $(\geq 0.5 \mathrm{~T} \mathrm{rms})$, low frequency $(\leq 50 \mathrm{~Hz})$ magnetic fields. The rationale behind this is the ready availability of strong permanent magnets and mains power for high strength electromagnets. This high field strength is needed to saturate the sample and compensate for the relatively low sensitivity of magnetic particle detection media, silicon Hall sensors, coils and other magnetic transducers used in such methods.
\end{abstract}

Consequently, frequencies greater than $50 \mathrm{~Hz}$ and applied magnetic fields less than 100 mT strength have not been widely explored for MFL due to the lack of commercially available sensors capable of detecting the leakage fields (typically in the nT and $\mu \mathrm{T}$ range) with adequate versatility to cope with the variations in inspection parameters, such as changes in lift-off, material properties, etc. which are inherent to NDT\&E settings.

In this study, the MFL response of surface-breaking longitudinal cracks from a ground mild steel weld validation sample, within the DC $-1 \mathrm{kHz}, 5 \mathrm{mT}-100 \mathrm{mT}$ rms applied magnetic field operating range, was explored. This was done to determine whether any optimal frequency response exists, better accommodating the inherent sample material properties (e.g. magnetic permeability and electrical conductivity), MFL mechanism and attributing phenomena such as electromagnetic skin effect and eddy current contributions.

Contrary to previous work published in Insight earlier this year, this study found no particular optimal frequency within this operating range, with explanations to justify the disproval of previously reported conclusions about optimal frequencies within this range. Also, the iteratively developed quantitative analysis performed in this study can be used to help further understand the underlying mechanisms of AC MFL and provide best practice regarding the optimisation of MFL. 


\section{Introduction}

Electromagnetic Non Destructive Testing and Evaluation (NDT\&E) techniques are often the preferred choice for surface-breaking flaw detection and evaluation in mild steel welds due to the versatility and large inspection area of Magnetic Particle Inspection (MPI) ${ }^{(1)}$, as well as the depth measurement and higher sensitivity of conventional Eddy Current Testing (ECT), due to the electromagnetic skin effect and other eddy current-based mechanisms ${ }^{(2)}$.

An examination of the typical frequencies of applied magnetic field used for low power surface-breaking flaw NDT\&E in ferromagnetic materials, depicted in Figure 1, shows a distinct gap between the MFL and eddy current-based techniques ${ }^{(2)}$, including Eddy Current Array (ECA) and Tangential Eddy Current Array (TECA). It must be noted that Alternating Current Field Measurement (ACFM) shown below is used to detect flaws in both ferromagnetic and paramagnetic materials ${ }^{(3)}$.

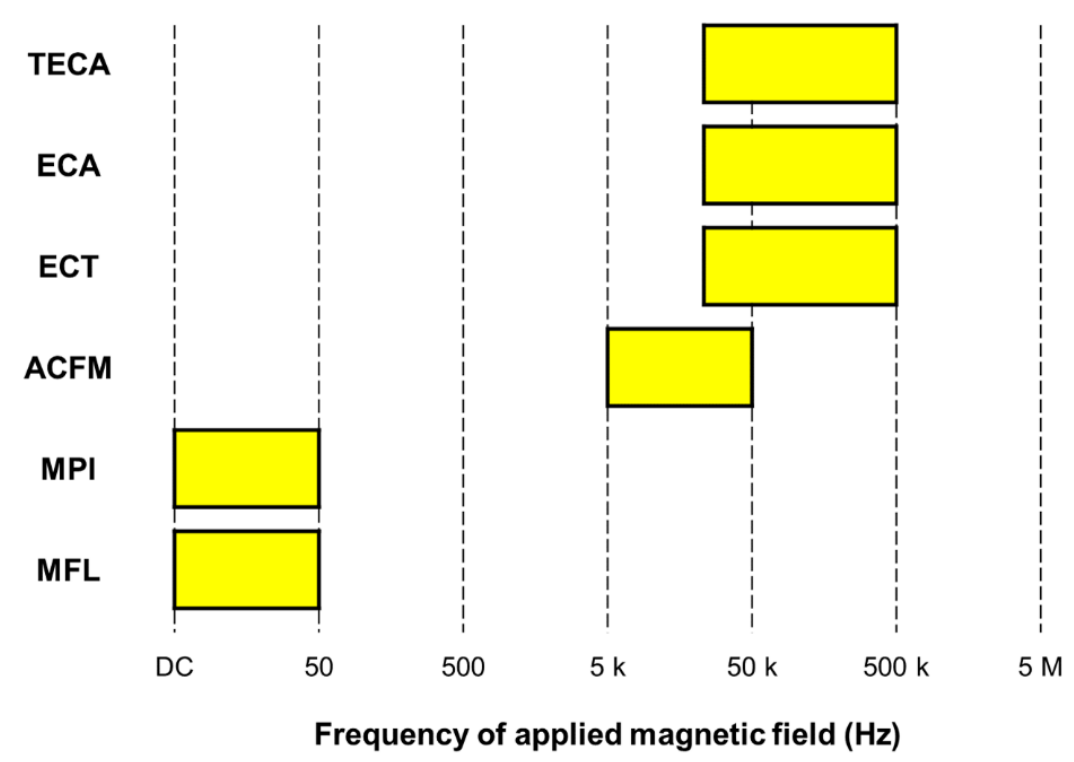

Figure 1. Typical range of applied magnetic field frequencies used for different lowpower electromagnetic methods for surface-breaking flaw NDT\&E in ferromagnetic materials.

The main reasons for this distinct gap include: higher frequencies generate more eddy current based on Faraday's Law; coil sensors are more sensitive at higher frequencies; some MFL-based methods use a DC field to achieve full sample penetration (no electromagnetic skin effect); coils, generic silicon Hall sensors and detection media are insensitive to low strength magnetic fields, so large applied field strengths are required (conveniently generated using $50 \mathrm{~Hz}$ mains).

Consequently, for low power surface-breaking flaw MFL-based methods, such as MPI and MFL imaging, an applied magnetic field frequency of $50 \mathrm{~Hz}$ is used out of convenience and necessity for the illuminating coil power consumption requirements because of detection insensitivity (transducer or media). 
Some exceptions to the range shown in Figure 1 above do exist, in particular high power $\sim 100 \mathrm{~kW} \sim 10-30 \mathrm{kHz}$, in situ MFL systems which do not require the portability for inservice inspection. These systems ${ }^{(4,5)}$ typically are very sensitive compared to other MFL techniques due to the use of a much higher frequency (electromagnetic skin effect) and high strength applied field for magnetic saturation within at least 1 skin depth. However, these systems also typically use detector coils, generic silicon Hall sensors and/or GMRs which all have their own problems relating to sensitivity, linearity or size (limited local resolution, array pitch, etc.).

This study focusses on the optimization of AC MFL for low power (i.e. portable) applications. As such, in this region the typical surface-breaking flaw MFL-based methods are not truly optimised to the magnetic response of the sample material; and instead are critically dependent on the sensitivity of the detection media / sensors used.

The QWHE sensors developed previously ${ }^{(6)}$ were used to ascertain if there was any optimum applied magnetic field frequency or field strength range for a particular type and size of flaws. The QWHE sensors were used because of their proven ability to detect the MFL response from real NDE flaws ${ }^{(7)}$. A previous comparative study ${ }^{(8)}$ (9) also suggested that they have a performance comparable to conventional ECT in controlled environment but with higher quality imaging capabilities (including additional weld information) and better flaw characterisation than eddy current-based images.

The key attributes of the QWHE sensors include: the ability to be made into bespoke arrays with specific sensor area $\left(2 \times 2 \mu \mathrm{m}^{2}\right.$ to $\left.70 \times 70 \mu \mathrm{m}^{2}\right)$ and sensor pitch $(<20 \mu \mathrm{m})$ allowing sensors to be optimised to particular NDT\&E applications, settings and requirements; sensitive to only one component of the sample magnetic field response, i.e. $B_{z}$ in Equation 1 below; high sensitivity which is limited by biasing and detection circuit electronics (currently $20 \mathrm{nTVHz}$ detection limit using superheterodyne biasing technique); wide operating range of $20 \mathrm{nT}$ to $2 \mathrm{~T}(160 \mathrm{~dB}), \mathrm{DC}$ to $\sim 50 \mathrm{MHz}$ range for versatility with no hysteresis offset unlike Giant Magnetoresistors (GMRs) and other anisotropic magnetoresistance-based transducers; linear across wide operating range leading to imaging capabilities of higher quality that are easier to process.

The Hall voltage $V_{H}$ created between a pair of sensing contacts is proportional to the biasing current, I, and the magnetic field component perpendicular to the device plate, $B_{Z}$ :

$V_{H}=\frac{1}{t \cdot n \cdot e} \cdot I \cdot B_{Z}$

Where $V_{H}$ is the Hall voltage, $t$ is the thickness of the 2DEG channel, $n$ is the electron concentration, $e$ is the electron charge, $I$ is the biasing current and $B_{z}$ is the component of magnetic field detected.

Equation (1) above emphasises that the thickness $t$ of the 2DEG channel and electron concentration $n$ determine the underlying sensitivity of the device, which have been developed over the past 20 years. 
It is the unique combination of sensitivity and linearity over a large operating range that makes QWHE sensors versatile for different NDT\&E applications, in particular, optimised low-power high-quality (flaw characterisation) MFL imaging.

It must be noted that QWHE sensors operate using the "conventional" Hall effect, as described above in Equation (1); and they do not use the Quantum Hall Effect or require active cooling like other Quantum-enabled transducers (i.e. SQUIDs). 


\section{Optimisation of Inspection Parameters for Low Power MFL Imaging}

Ground mild steel plate weld samples manufactured by Sonaspection ${ }^{(10)}$, with two surface-breaking longitudinal toe cracks of lengths $10 \mathrm{~mm}, 11 \mathrm{~mm}$ and $8 \mathrm{~mm}$, were used in this study. Figure 2 below shows simplified versions of the drawings supplied by Sonaspection and a diagram to explain the artefacts in the resultant magnetic images:

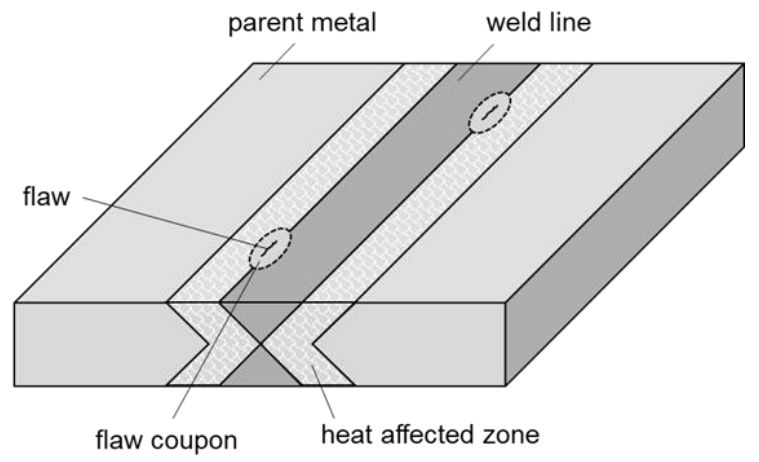

(a) typical sample layout

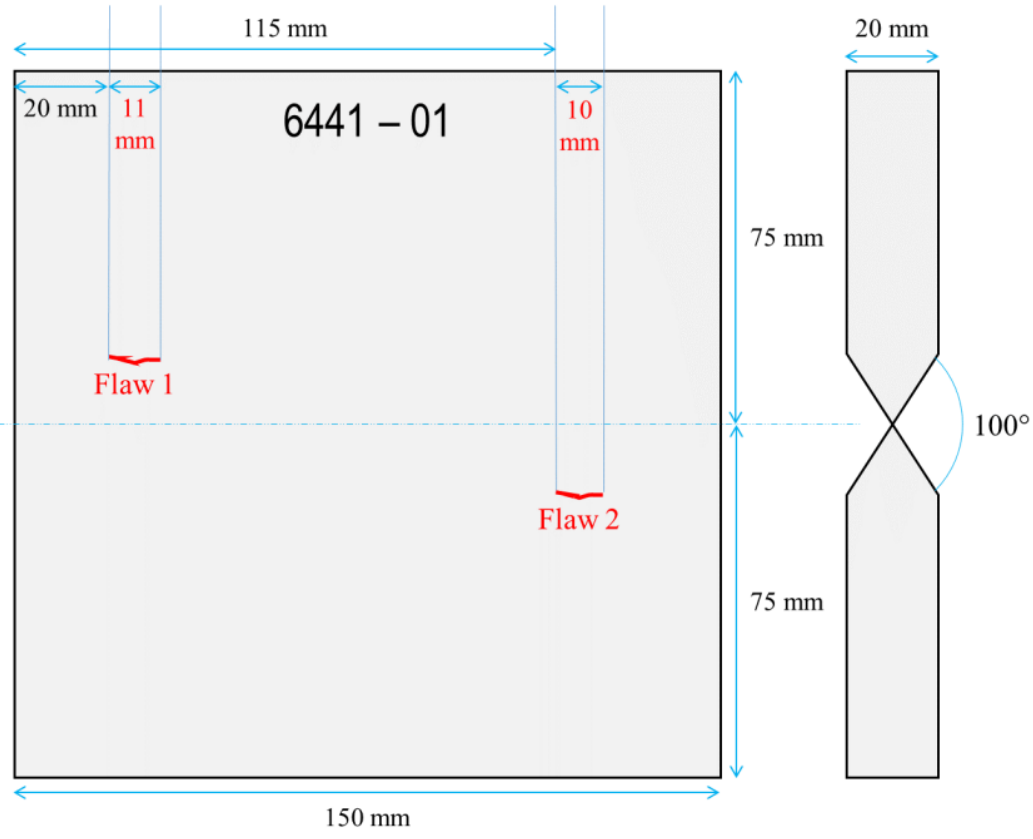

(b) Sample 6441-01 (11 mm and $10 \mathrm{~mm}$ flaws) 


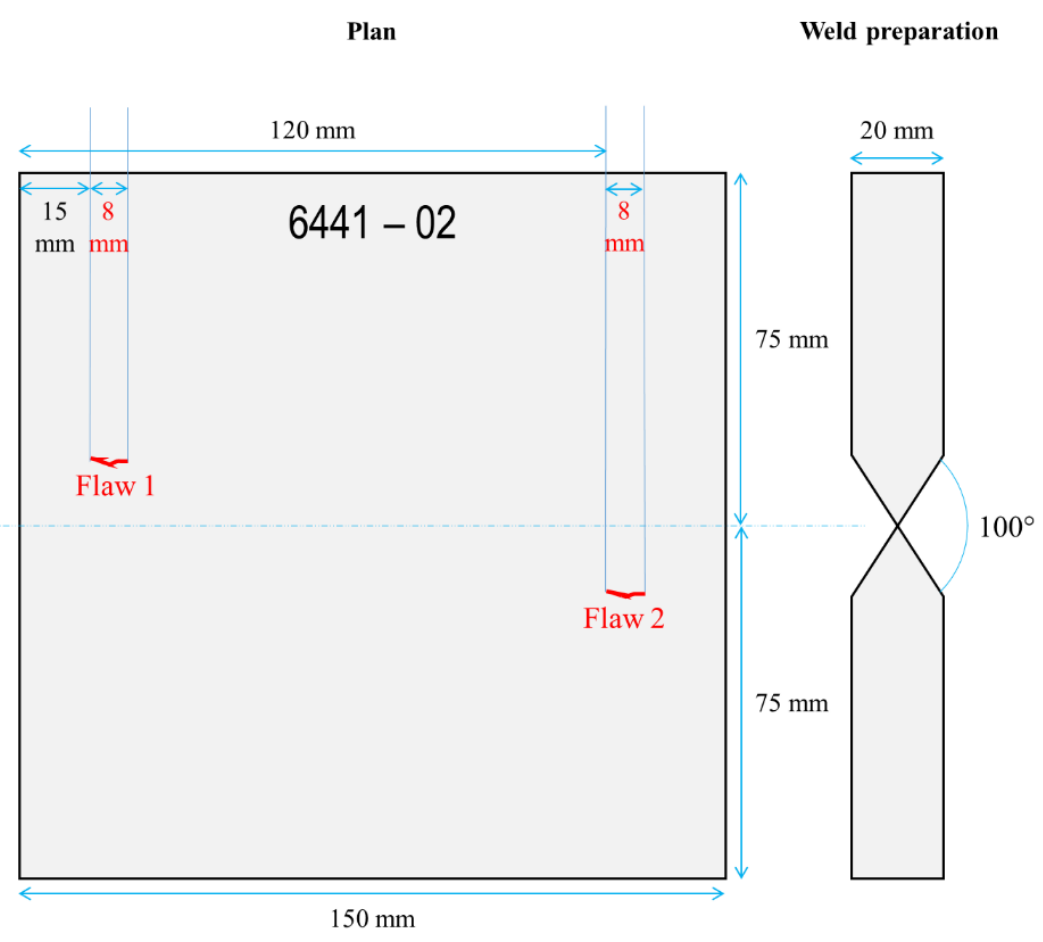

(c) Sample 6441-02 (8 mm flaws)

Figure 2. Simplified sample drawings from specimen manufacturer.

As Figure 2 shows, the samples measured $150 \mathrm{~mm} \times 150 \mathrm{~mm}$ with a thickness of 20 $\mathrm{mm}$. They contained a full penetration weld whose cap and root were ground flush. The parent metal was generic mild steel, with electrical and magnetic properties similar to AISI 1010. MPI was used to validate the sizes of the flaws, however a previous study found that QWHE magnetic imaging, conventional ECT and ACFM values differed slightly (all within a $\pm 2 \mathrm{~mm}$ range) ${ }^{(8,9)}$. For this study, the MFL response from the two toe cracks on each sample were investigated.

The developed scanner reported previously (11) (12) autonomously controlled the fine movement of a probe consisting of a $10 \mu \mathrm{m}$ size QWHE sensor and its biasing/detection circuitry, along with an illuminating electromagnet. The electromagnet applied a magnetic field of controlled frequency and field strength to the sample, with the QWHE sensor and data acquisition system mapping the MFL response for each $x$ and $y$ position on the sample surface.

A proximity laser on the probe was used to take an initial topographical scan of the region to be magnetically imaged. An additional $z$ direction motor module was used to autonomously control the probe lift-off, using the laser map to compensate for changes in lift-off due to sample curvature. This was done to avoid damage to the probe head and sample, as well as provide better quality magnetic images.

A previously reported study ${ }^{(12)}$ used Sample 6441-01 which was scanned using applied magnetic field frequencies and field strengths across the entire operational range of the 
QWHE magnetic imaging system (limited by the illuminating coil parameters). This range is shown in Figure 3 below:

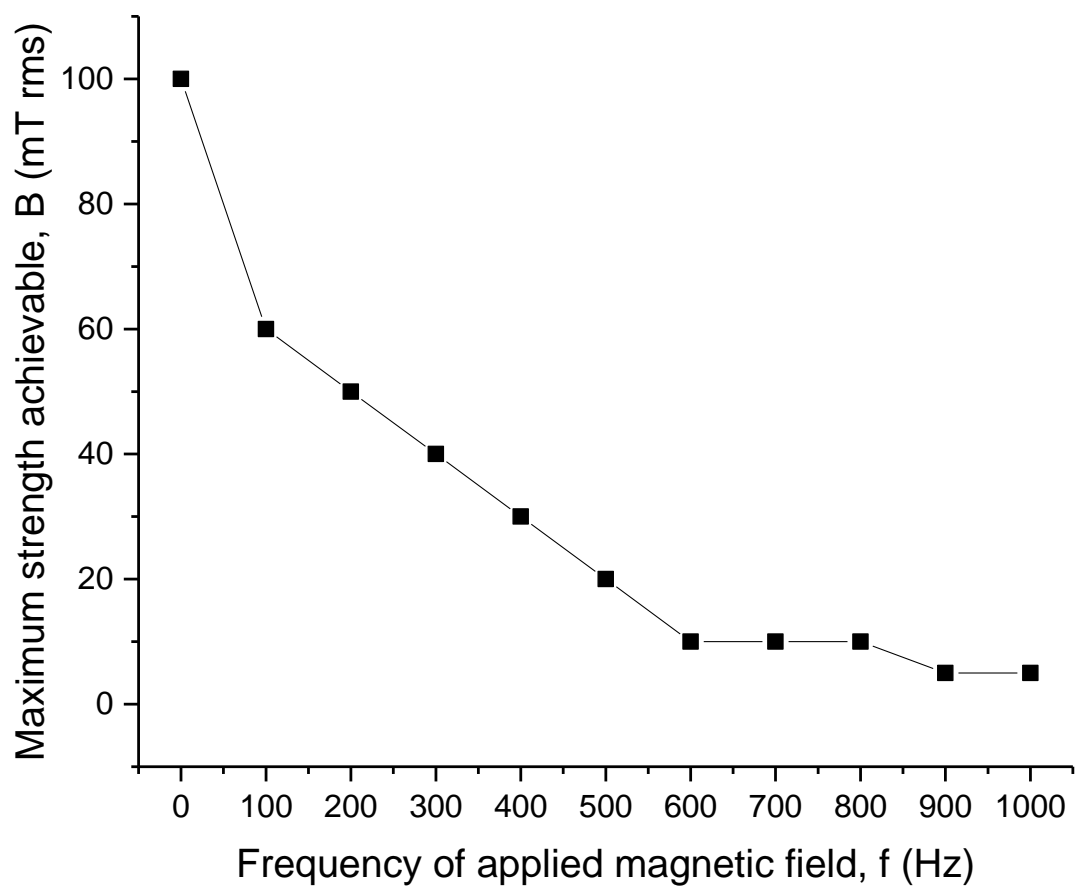

Figure 3. Plot showing the operational range of the XYZ scanner and the maximum strengths of applied magnetic field for different frequencies used in this work.

The sample was firmly secured to the scanner bed to ensure no movement during the scans (particularly at DC where strong field strengths can pick up the sample). A fine measurement step of $100 \mu \mathrm{m}$ for $x$ and $y$ was used (giving a pixel size of $100 \mu \mathrm{m} \times 100$ $\mu \mathrm{m})$, with a $0.75 \mathrm{~mm}$ lift-off to ensure adequate clearance throughout the scanning.

As shown in Figure 4 below, a region of $70 \mathrm{~mm}$ (approximately central across the weld) $\mathrm{x} 140 \mathrm{~mm}$ (along the weld) was scanned, with the regions identified used for quantitative analysis: 


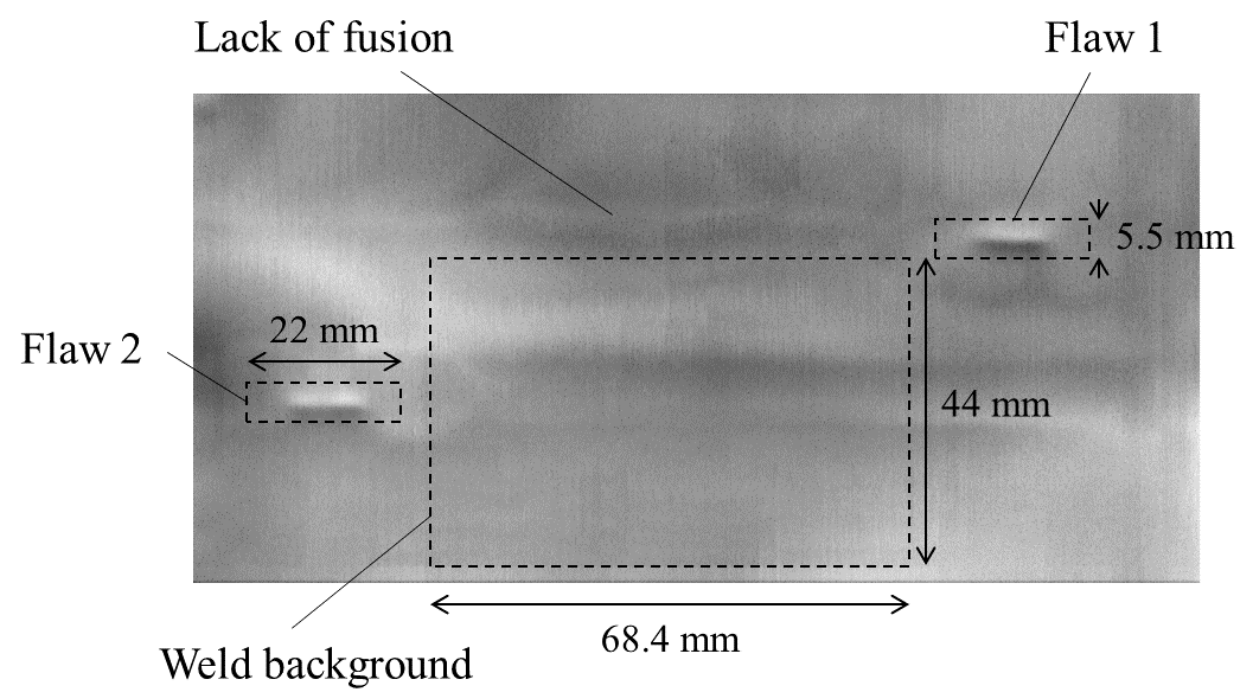

Figure 4. Example raw absolute magnetic image showing regions of interest used for quantitative analysis.

Differential pixel values $\boldsymbol{j}_{\boldsymbol{f} \boldsymbol{B} \boldsymbol{x} \boldsymbol{y}}$, and subsequent differential images, were obtained by performing a basic differential filter (in the direction of the applied magnetic field) on the acquired absolute data $\zeta_{\boldsymbol{f} \boldsymbol{B} \boldsymbol{x} \boldsymbol{y}}$, as shown below in Equation (2):

$j_{f B x y}=\frac{\zeta_{f B x y}-\zeta_{f B\left(x-x^{\prime}\right) y}}{x}$

Where $\boldsymbol{j}_{\boldsymbol{f} \boldsymbol{B} \boldsymbol{x} \boldsymbol{y}}$ is the differential pixel value for that $(\boldsymbol{x}, \boldsymbol{y})$ position $\left(\mathrm{mT} \mathrm{mm}^{-1}\right)$ for applied magnetic field of frequency $\boldsymbol{f}(\mathrm{Hz})$ and strength $\boldsymbol{B}(\mathrm{mT} \mathrm{rms}), \boldsymbol{\zeta}_{\boldsymbol{f} \boldsymbol{B} x \boldsymbol{y}}$ is the raw absolute (mT) pixel value for position $(\boldsymbol{x}, \boldsymbol{y}), \boldsymbol{x}$ is the direction of the applied magnetic field and the probe velocity and $\grave{x}$ is the $\boldsymbol{x}-\boldsymbol{y}$ measurement step (mm).

The impact of using differential imaging over absolute is emphasised below in Figure 5 with a few examples of magnetic images obtained from these scans (note the clarity and prominence of the flaws in differential mode):

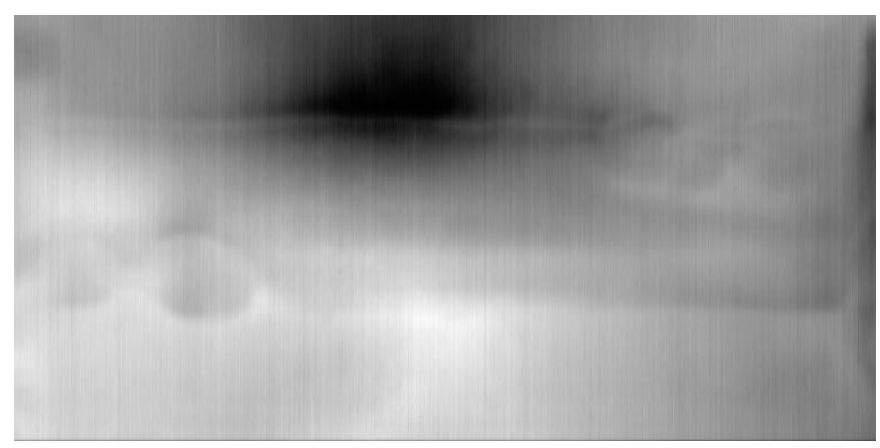

(a) Absolute DC $40 \mathrm{mT}$ image 
(b) Differential DC $40 \mathrm{mT}$ image

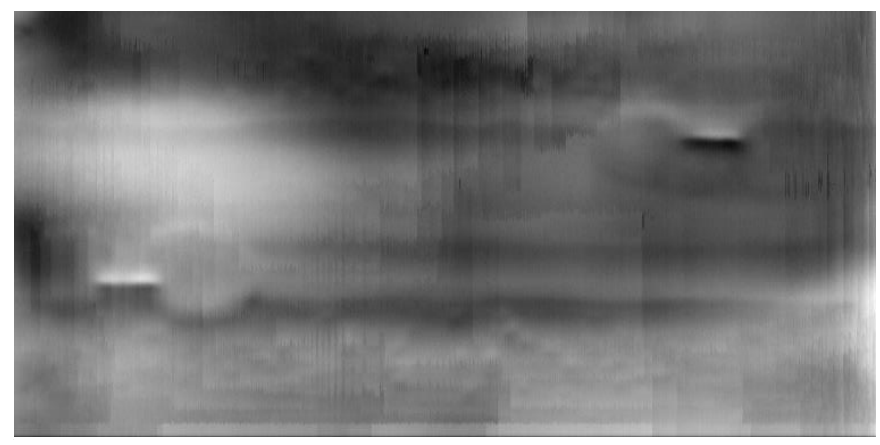

(c) Absolute $500 \mathrm{~Hz} 20 \mathrm{mT}$ image

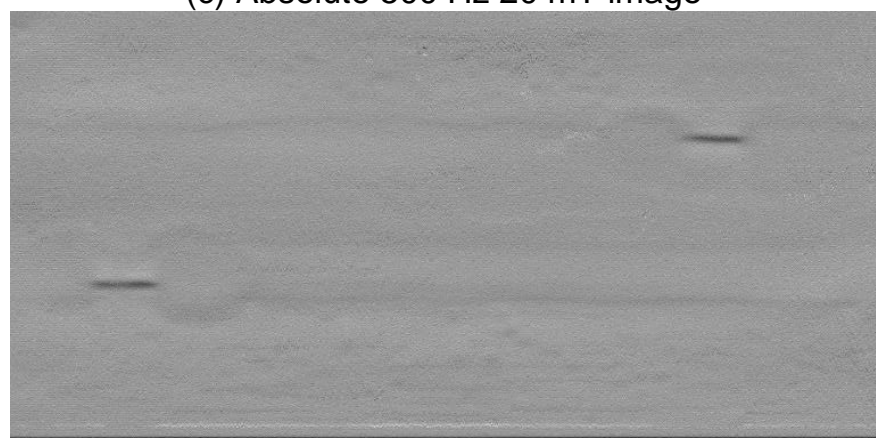

(d) Differential $500 \mathrm{~Hz} 20 \mathrm{mT}$ image

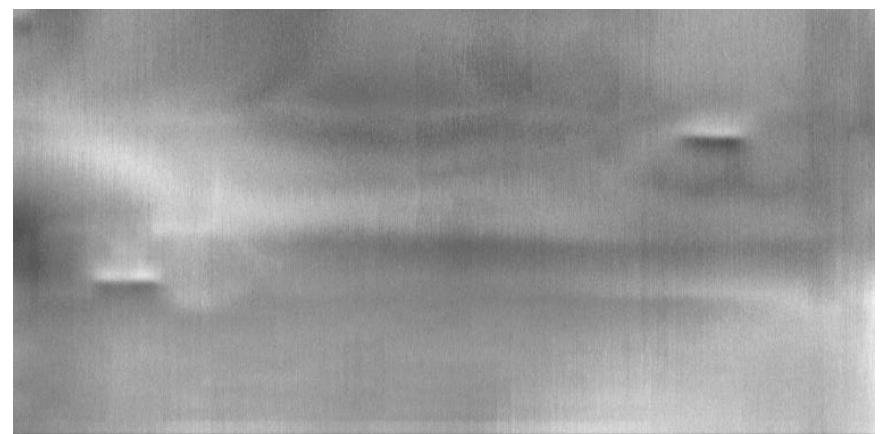

(e) Absolute $1 \mathrm{kHz} 5 \mathrm{mT}$ image 
(f) Differential $1 \mathrm{kHz} 5 \mathrm{mT}$ image

Figure 5. Examples of some absolute and differential magnetic images from some of the scans of Sample 6441-01.

Each raw unprocessed differential magnetic image, for the range of magnetic field frequency and field strength scans, was quantitatively analysed to determine whether an optimal range with higher flaw detectability was present. This was achieved by calculating the rms pixel value $\gamma$ for each of the three regions of interest shown in Figure 4 above: Flaw 1, Flaw 2 and the background weld (i.e. $i=1,2,0$ respectively). This calculation was extracted from Equation (3) below:

$\gamma_{i f B}=\sqrt{\frac{\grave{x}^{2}}{\left(x_{\max }-x_{\min }\right)\left(y_{\max }-y_{\min }\right)} \int_{x_{\min }}^{x_{\max }} \int_{y_{\min }}^{y_{\max }}\left(j_{f B x y}\right)^{2} \mathrm{dy} \mathrm{dx}}$

$\beta_{i f B}=20 \log \left(\frac{\gamma_{i f B}}{\gamma_{0 f B}}\right)$

$\bar{\beta}_{f B}=20 \log \left(\frac{1}{2} \frac{\gamma_{1 f B}+\gamma_{2 f B}}{\gamma_{0 f B}}\right)$

Where $\boldsymbol{\gamma}_{\boldsymbol{i} \boldsymbol{B} \boldsymbol{B}}$ is the rms value $\left(\mathrm{mT} \mathrm{mm}^{-1}\right)$ for that region $\boldsymbol{i}$ of interest, $\boldsymbol{\beta}_{\boldsymbol{i f} \boldsymbol{B}}$ is the flaw $\mathrm{SNR}(\mathrm{dB})$, i.e. ratio of MFL response from flaw and weld background; and $\overline{\boldsymbol{\beta}}_{\boldsymbol{f} \boldsymbol{B}}$ is the mean flaw SNR $(\mathrm{dB})$.

These SNR values previously reported ${ }^{(12)}$ are shown below in Figure 6 with an estimated error of $\pm 10 \%(+0.828 \mathrm{~dB}$ and $-0.915 \mathrm{~dB})$ based on the two quantities used in the calculation, both containing an estimated 5\% error originating from the XYZ scanner measurements: 


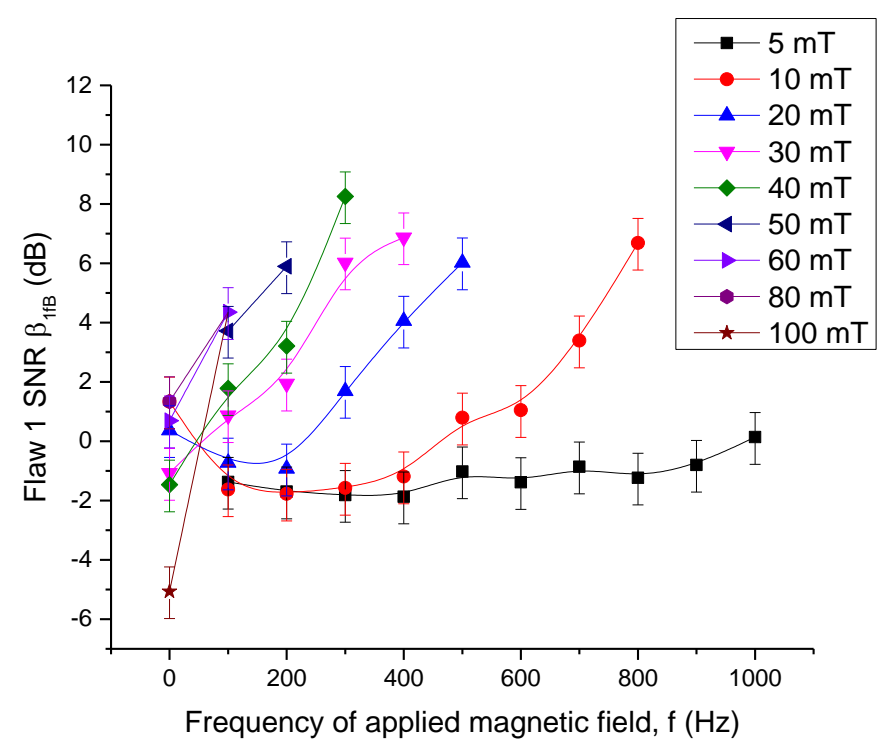

(a) SNR plot for Flaw 1

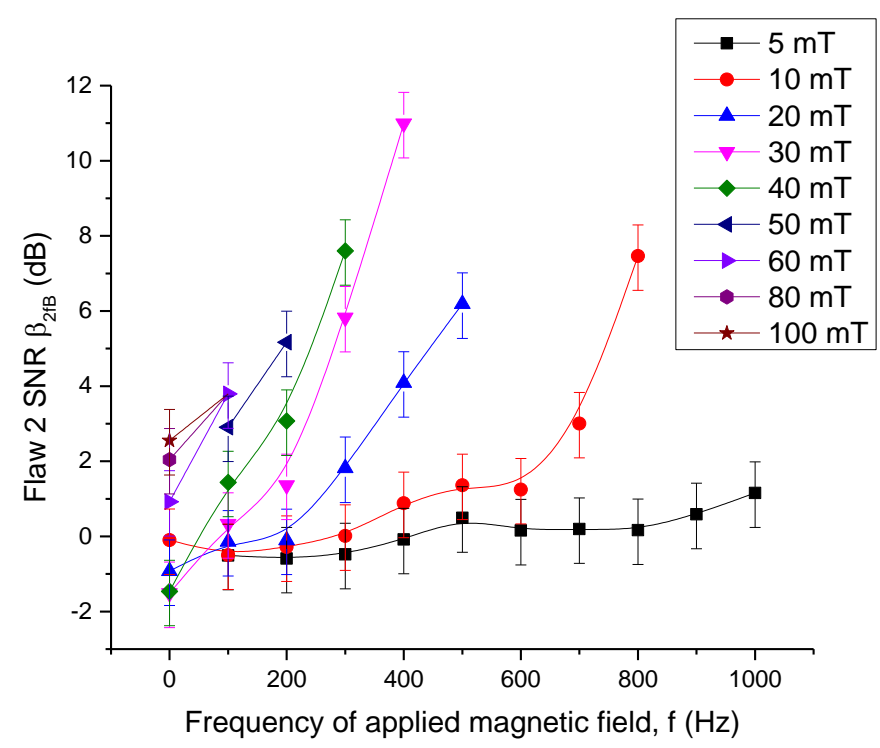

(b) SNR plot for Flaw 2 


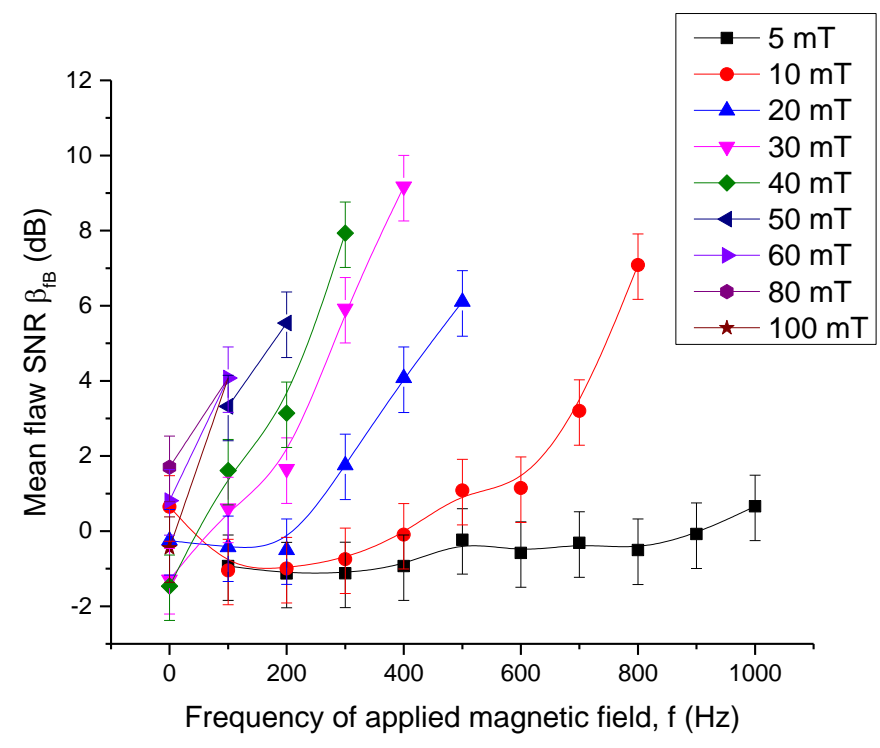

(c) Mean SNR plot

Figure 6. SNR plots obtained from scans of Sample 6441-01.

These plots show a definitive increase in flaw detectability using higher frequencies and stronger strength applied magnetic fields, adding confidence to the quantitative analysis process. Previously it was reported that these plots suggest an inflection point, where the SNR begins to dramatically increase, at approximately $f \geq 200 \mathrm{~Hz}^{(12)}$, with conjecture regarding the root cause being due to either the magnetic properties of the sample material (AISI 1010) or due to the electromagnetic skin effect, or a combination of both. This was because changes in microstructure or parent metal magnetic permeability due to higher frequency (i.e. magnetic relaxation) would result in less MFL from microstructure-metal boundaries, producing less background weld noise, increasing flaw SNR. Alternatively, higher frequencies of applied magnetic field are confined closer to the sample surface (i.e. electromagnetic skin effect), meaning that there is less perturbation from microstructures within the volume of the sample, producing less background noise and thus increasing flaw SNR. The electromagnetic skin effect could also be responsible since higher frequencies of applied magnetic field are confined closer to the sample surface, meaning higher strength magnetic field at flaw boundary, producing more MFL flaw signal and increasing flaw SNR.

To investigate the root cause of this inflection point phenomena, the study was repeated using a second specimen, Sample 6441-02, which contained smaller flaws of length 8 $\mathrm{mm}$ and smaller depths (estimated as $>5.6 \mathrm{~mm}$ for Sample 6441-01 and > $3.5 \mathrm{~mm}$ using ACFM in previous work ${ }^{(8,9)}$ ) which was scanned under the same controlled conditions and inspection parameters as the previous study. This sample was prepared at the same time as 6441-01 using the same parent and weld metals, the same welding technique and parameters, and was performed by the same welder. As such, the inspection parameters were controlled and kept as similar as possible for both specimens and both investigations. 
Based on the root cause theories made above, the inflection point would either remain constant or increase, depending on the cause of the phenomena. As such, the theory involving magnetic relaxation would produce no change in inflection point since it can be assumed that on average the microstructures in Sample 6441-01 resemble those of 6441-02. Likewise, the theory involving the electromagnetic skin effect reducing the background noise due to detecting fewer microstructures would result in no change. However, the explanation involving the electromagnetic skin effect creating stronger MFL responses from flaws because of the stronger applied magnetic fields at flaw boundaries due to increased confinement would produce an increase in inflection point frequency since the flaws on Sample 6441-02 were $~ 38 \%$ less deep than those in Sample 6441-01. 


\section{Results and Discussion}

For consistency, the resultant images obtained for Sample 6441-02 which correspond to those shown in Figure 5, are shown below in Figure 7. As before, note the clarity and prominence of the flaws in differential mode:

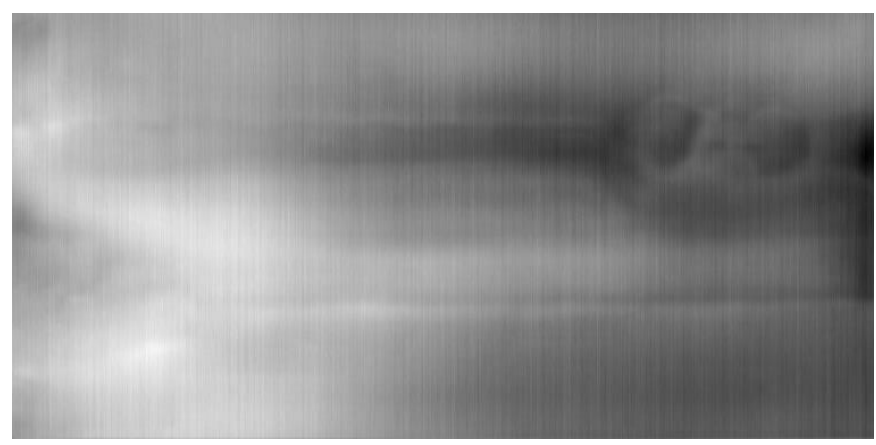

(a) Absolute DC $40 \mathrm{mT}$ image

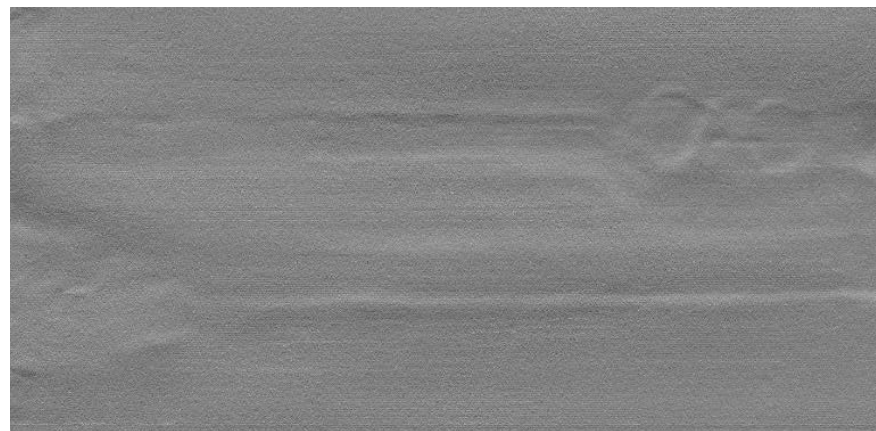

(b) Differential DC $40 \mathrm{mT}$ image

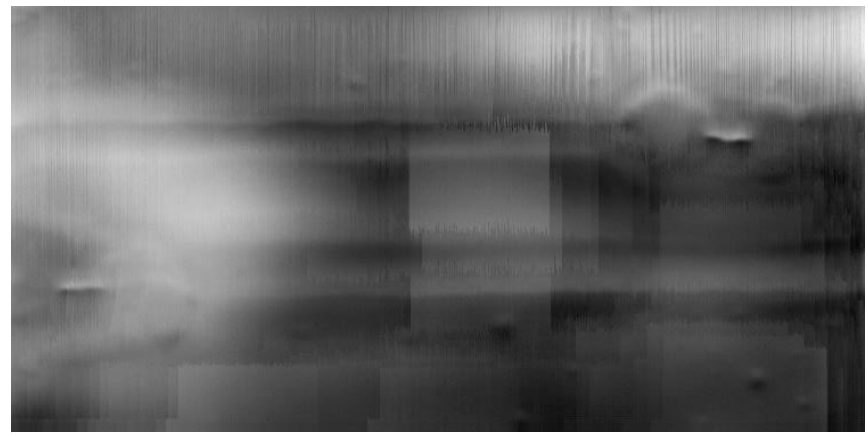

(c) Absolute $500 \mathrm{~Hz} 20 \mathrm{mT}$ image

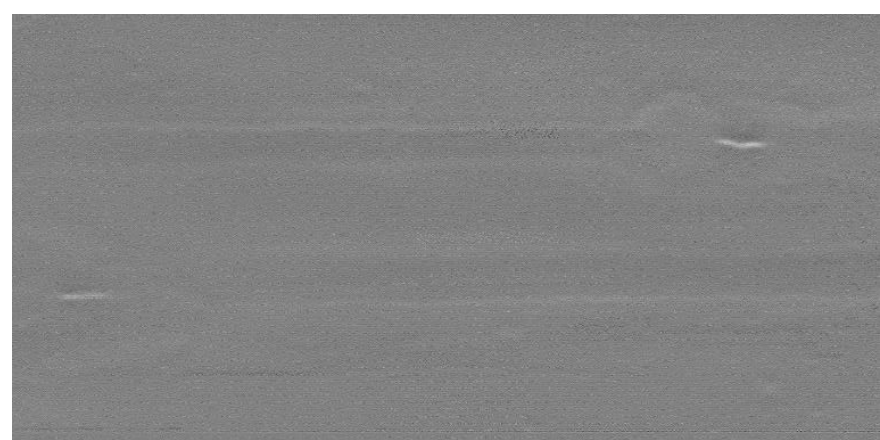

(d) Differential $500 \mathrm{~Hz} 20 \mathrm{mT}$ image 
(e) Absolute $1 \mathrm{kHz} 5 \mathrm{mT}$ image

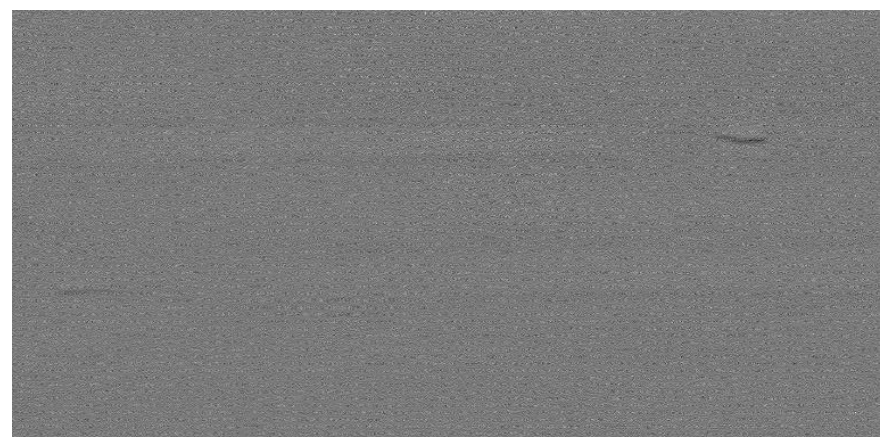

(f) Differential $1 \mathrm{kHz} 5 \mathrm{mT}$ image

\section{Figure 7. Corresponding examples of some absolute and differential} magnetic images from some of the scans of Sample 6441-02.

To test the origin of this inflection point, the same quantitative analysis was performed on the differential images obtained for Sample 6441-02, with SNR plots shown below in Figure 8.

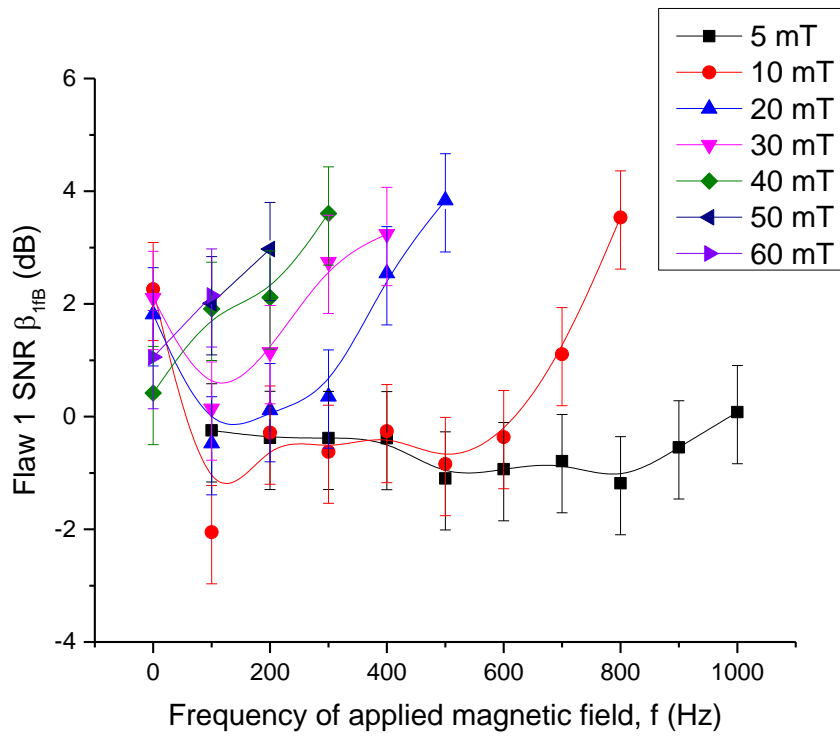

(a) SNR plot for Flaw 1 


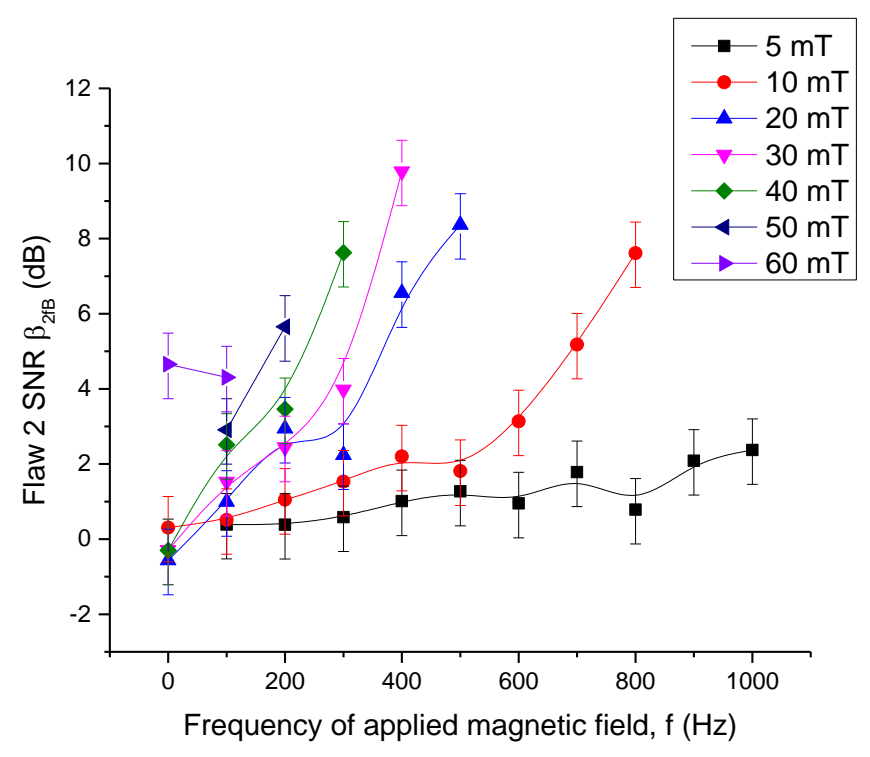

(b) SNR plot for Flaw 2

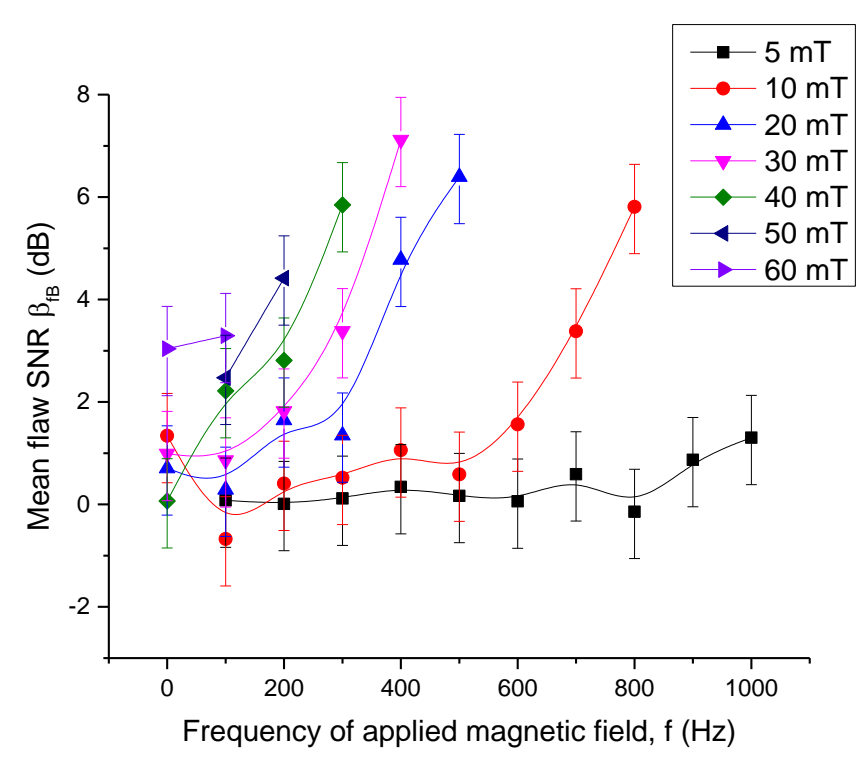

(c) Mean SNR plot

Figure 8. SNR plots obtained from scans of Sample 6441-02.

Figure 8 also shows that flaw detectability is increased when higher frequencies and stronger strengths of applied magnetic field are used, agreeing with the previous investigation and adding confidence to the quantitative analysis performed.

However, Figure 8 does also suggest a reduction in inflection point frequency which was not predicted, suggesting that the phenomena is not related to changes in magnetic permeability (i.e. magnetic relaxation) of microstructures / parent metal within this DC $1 \mathrm{kHz}$ operating range or from the electromagnetic skin effect. 
To further investigate whether this inflection point was related to electromagnetic skin effect, the B-H curve for AISI 1010 was used to transform the B-field applied magnetic field strength into quasi-H field. This was considered a quasi-quantity since the calibrations were obtained with some degree of lift-off involved, i.e. taken in air which does not have a B-H curve of AISI 1010. The strength of the applied magnetic field was also estimated using Equation (4) below:

$H_{d}=H_{0} e^{-\frac{d}{\delta}}$

$$
\delta=\sqrt{\frac{1}{\pi f \mu_{o} \mu_{r} \sigma}}
$$

Where $\boldsymbol{H}_{\boldsymbol{d}}$ is approximated strength of the applied magnetic field at the flaw depth (A $\left.\mathrm{m}^{-1}\right), \boldsymbol{H}_{\mathbf{0}}$ is the strength of the applied magnetic field transformed into its quasi-H quantity $\left(\mathrm{A} \mathrm{m}^{-1}\right), \boldsymbol{d}$ is the flaw depth $(\mathrm{mm}), \boldsymbol{\delta}$ is the skin depth $(\mathrm{m}), \boldsymbol{f}$ is the frequency of the applied magnetic field, $\boldsymbol{\mu}_{\boldsymbol{o}}$ is the magnetic permeability of free space, $\boldsymbol{\mu}_{\boldsymbol{r}}$ is the relative magnetic permeability of the sample and $\boldsymbol{\sigma}$ is the electrical conductivity of the sample $\left(\mathrm{S} \mathrm{m}^{-1}\right)$.

Using this, the mean SNR plots for Sample 6441-01 (i.e. from Figure 6) were transformed as shown below in Figure 9:

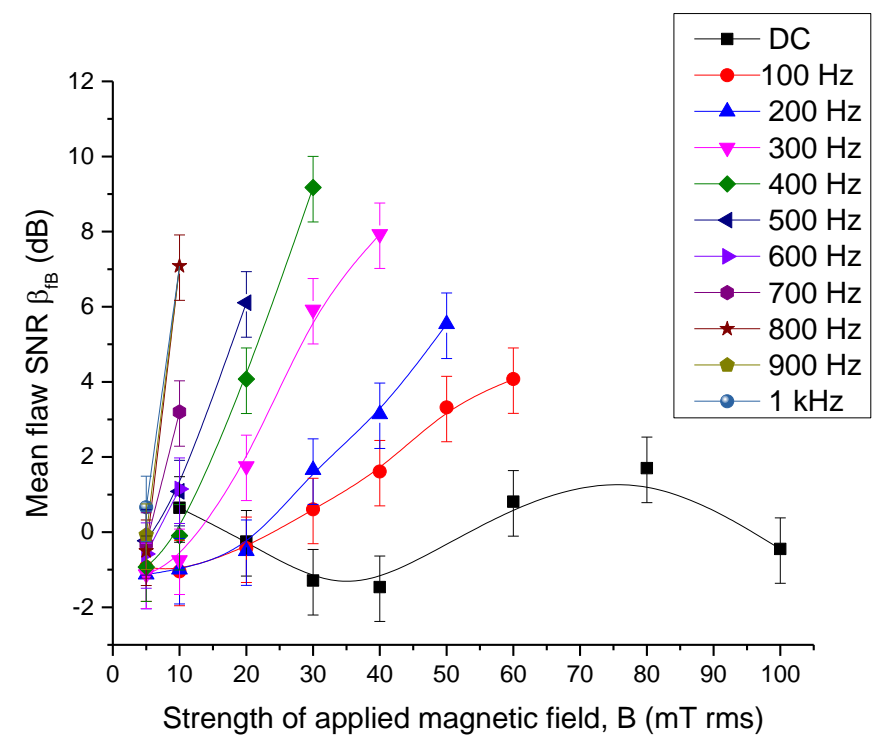

(a) Mean SNR plot with no B-H transformation for comparison 


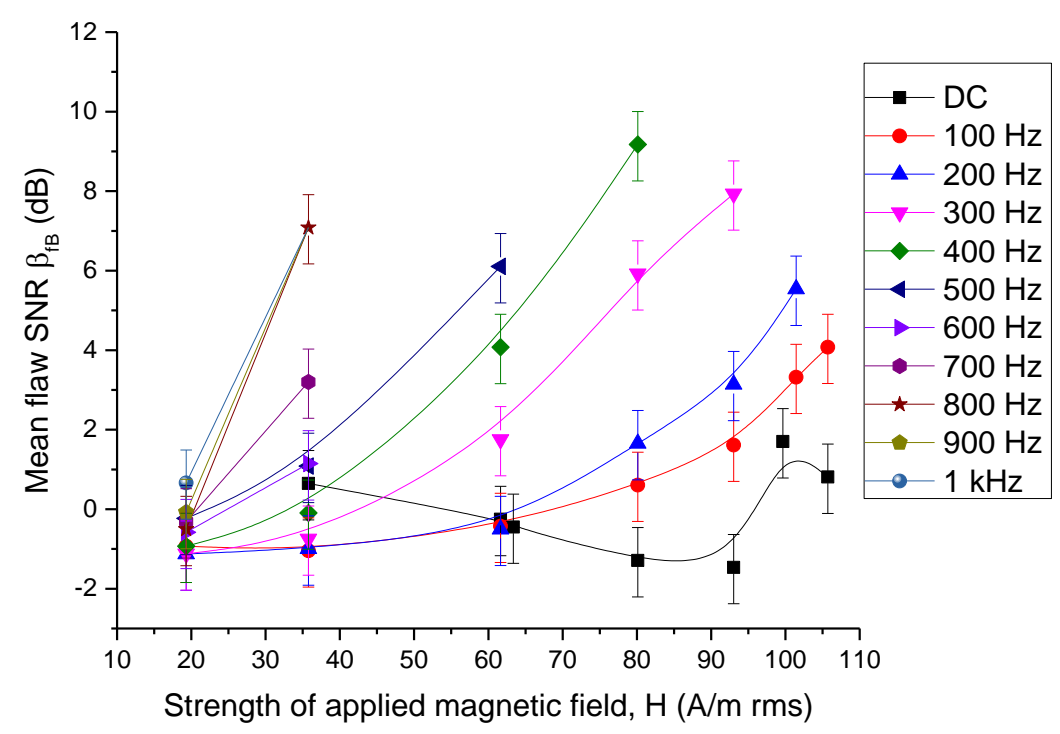

(b) Mean SNR plot with applied magnetic field transformation

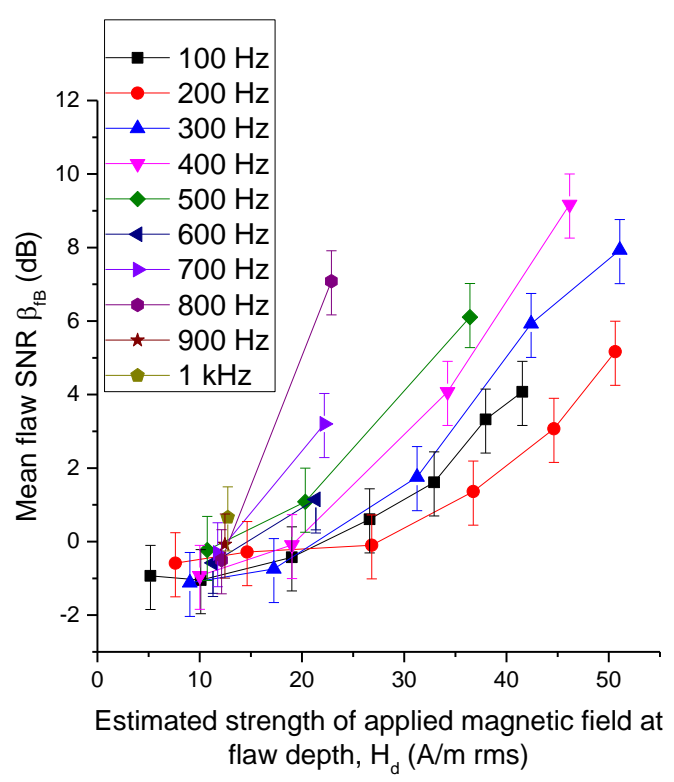

(c) Mean SNR plot with applied magnetic field transformation and estimation of field at sample depth

\section{Figure 9. SNR plots using transformed applied magnetic field} obtained from scans of Sample 6441-01.

Figure 9(a) shows that there is a clear distinction between the trends for SNRs with frequencies $>200 \mathrm{~Hz}$, indicative of the inflection point phenomena. However, even with approximate estimation of the applied magnetic field in $\mathrm{H}$-field, this distinction has been lessened; and with coarse estimation of field strength at the flaw depth the distinction has been completely removed.

As such, this analysis suggests that the inflection point phenomenon is simply an artefact of B-H non-linearity and electromagnetic skin effect, where the low frequency strongest 
applied magnetic fields were less represented on B-field vs. SNR plots than on $\sim \mathrm{H}$-field vs. SNR, and confirms that the applied magnetic field used in this AC MFL technique is affected by the electromagnetic skin effect (even though it is applied to and not induced in the sample).

Therefore, this work has emphasised the importance and best practice to convert applied magnetic fields into quasi-H field quantities for fairer representation when analysing for optimisation and flaw detectability. It therefore also suggests it is good practice to convert MFL measurements into quasi-H field quantities too.

This work also shows that there is no particular "optimum" applied magnetic field or frequency within the operational range of the XYZ scanner, DC $-1 \mathrm{kHz}, 5 \mathrm{mT}-100 \mathrm{mT}$ rms applied field, for MFL performed using an electromagnet producing such a magnetic field behaviour, instead showing that flaw detectability is simply increased using the highest frequency achievable of sufficient strength (which can be determined using this optimisation procedure). 


\section{Conclusions}

In this work, magnetic images using a QWHE sensor XYZ scanner were obtained with various applied magnetic field frequency and field strengths across the complete operational range of the scanner (DC $-1 \mathrm{kHz}, 5 \mathrm{mT}-100 \mathrm{mT}$ rms applied field).

Quantitative analysis was then performed to determine that there is no optimum applied magnetic field frequency or field strength determined by the sample material properties within the operational range of the XYZ scanner. This work details the iterative development and optimisation of the low power MFL imaging technique and outlines some best practice procedures regarding inspection parameter optimisation.

It is intended that this work is continued with the development of a new electromagnet for the XYZ scanner which is capable of higher frequencies of applied magnetic field strength within the $\geq 5 \mathrm{mT}$ rms region, to determine if such an optimum frequency exists, better suited to the inherent material properties or nature of MFL. It is also intended to use this data, along with additional investigations, to help develop the underlying knowledge of AC MFL mechanisms towards a simple low-strength $(<100 \mathrm{mT} \mathrm{rms}$ applied field) dipole model accounting for AC phenomena.

Future work being undertaken to develop the maturity of the QWHE imaging technique includes: repeating the study using various lift-off values to measure the effect of lift-off for MFL imaging using QWHE sensors; developing enhanced, optimised imaging technology based on this underpinning research; developing image enhancement techniques and automated flaw detection and sizing algorithms using frequency-based analysis and/or spatial MFL field distribution around flaws; building a database of MFL responses from flaws of known dimensions for future enhanced characterisation and reconstruction; and developing multi-frequency / pulsed applied magnetic field technologies for detection, imaging, sizing and characterisation of surface-breaking and near-surface flaws $(<3 \mathrm{~mm}$ from surface) for ferromagnetic and non-ferrous conductive materials (i.e. titanium alloys and carbon fibre composites).

\section{Acknowledgements}

EPSRC-EP/P006973/1 "FUTURE COMPOUND SEMI-CONDUCTOR MANUFACTURING HUB”)

EPSRC-EP/LO22125/1 "UK RESEARCH CENTRE IN NON-DESTRUCTIVE EVALUATION(RCNDE3)"

ICASE PhD STUDENTSHIP THROUGH RCNDE (BAE Systems) 


\section{References}

1. British Standard BS EN ISO 9934-1:2016. Non-destructive testing - Magnetic particle testing. Part 1: General principles (ISO 9934-1:2016), 2016.

2. S. S. Udpa, 'NDT Handbook - Electromagnetic Testing' (3rd ed.). Columbus: American Society for Nondestructive Testing, 2004.

3. R. E. Beissner, C. M. Teller, G. K. Burkhardt, R. T. Smith and J. R. Barton, 'Detection and Analysis of Electric-Current Pertubation Caused by Defects', EddyCurrent Characterization of Materials and Structures, ed. G. Birnbaum and G. Free (West Conshohocken, PA: ASTM International, 1981), 428-446. DOI 10.1520/STP27602S.

4. British Standard BS EN ISO 15549:2010. Non-destructive testing - Eddy current testing - General principles, 2010.

5. https://www.foerstergroup.de/de/deu/

6. N. Haned and M. Missous, 'Nano-tesla magnetic field magnetometry using an InGaAs-AlGaAs-GaAs 2DEG Hall sensor', Sensors and Actuators, 102(3), 216222. DOI 10.1016/S0924-4247(02)00386-2, 2003.

7. J. M. Watson, C.W. Liang, E. Ahmad, J. Sexton and M. Missous, 'Surface Crack Detection in Dressed Steel Welds Using Advanced Quantum Well Hall Effect Sensors', 56th Annual Conference of the British Institute of Non-Destructive Testing, 2017. https://www.researchgate.net/publication/337947985_Surface_Crack_Detection_i n_Dressed_Steel_Welds_Using_Advanced_Quantum_Well_Hall_Effect_Sensors

8. J. M. Watson, C.W. Liang, J. Sexton, F. A. Biruu and M. Missous, 'A Comparative Study of Electromagnetic NDE Methods and Quantum Well Hall Effect Sensor Imaging for Surface-Flaw Detection in Mild Steel Welds', 57th Annual British Conference on Non-Destructive 2018. https://www.researchgate.net/publication/337947902_A_Comparative_Study_of_ Electromagnetic_NDE_Methods_and_Quantum_Well_Hall_Effect_Sensor_Imagi ng_for_Surface-Flaw_Detection_in_Mild_Steel_Welds

9. J. M. Watson, C.W. Liang, J. Sexton, F. A. Biruu and M. Missous, 'SurfaceBreaking Flaw Detection in Ferritic Welds using Quantum Well Hall Effect Sensor Devices', 45th Annual Review of Progress in Quantitative Nondestructive Evaluation. DOI 10.1063/1.5099818, 2018.

10. https://sonaspection.com/

11. C. W. Liang, 'A High Spatial Resolution Magnetovision Camera Using HighSensitivity Quantum Well Hall Effect Sensors', The University of Manchester, 2017. https://ethos.bl.uk/OrderDetails.do?uin=uk.bl.ethos.728031 
12. J. Watson, C. W. Liang, J. Sexton and M. Missous, 'Magnetic field frequency optimisation for MFL imaging using QWHE sensors', Insight, 62(7), July 2020, DOI 10.1784/insi.2020.62.7.396. 